\title{
Linx
}

Revue des linguistes de l'université Paris X Nanterre

$44 \mid 2001$

Spécificité et histoire des discours sémiotiques

\section{La sémiologie selon L. J. Prieto}

Sémir Badir

\section{OpenEdition}

\section{Journals}

Édition électronique

URL : http://journals.openedition.org/linx/1034

DOI : 10.4000/linx.1034

ISSN : 2118-9692

\section{Éditeur}

Presses universitaires de Paris Nanterre

\section{Édition imprimée}

Date de publication : 1 juin 2001

Pagination : $55-73$

ISSN : 0246-8743

\section{Référence électronique}

Sémir Badir, « La sémiologie selon L. J. Prieto », Linx [En ligne], 44 | 2001, mis en ligne le 05 juillet 2012, consulté le 19 avril 2019. URL : http://journals.openedition.org/linx/1034 ; DOI : 10.4000/linx.1034

Ce document a été généré automatiquement le 19 avril 2019

Département de Sciences du langage, Université Paris Ouest 


\title{
La sémiologie selon L. J. Prieto
}

\author{
Sémir Badir
}

1 Comment présenter Luis J. Prieto à son avantage ? Déclarerais-je qu'il a été, avec Roland Barthes, un pionnier de la sémiologie? Dès 1964, paraissaient ses Principes de noölogie, exposé aussi succinct qu'ambitieux d'une sémantique formelle ${ }^{1}$. En 1966, deux ans après les "Éléments de sémiologie» de Barthes, Prieto publia Messages et signaux, ouvrage d'introduction générale à la sémiologie. Il fut également chargé de rédiger l'article « La sémiologie » pour le volume Le Langage dans l'Encyclopédie de La Pléiade (paru en 1968). Nommé professeur de linguistique générale à Genève ${ }^{2}$, il devenait en quelque sorte l'exécuteur testamentaire de Saussure, appelé à réaliser la sémiologie que ce dernier avait tant méditée. Et sans doute, par ses ouvrages, Prieto réalisa-t-il une partie considérable de ce projet. Pourtant, dans l'histoire de la discipline, comme dans l'histoire générale des sciences humaines, son nom est rarement cité.

2 Rappellerais-je alors qu'il a été, avec Greimas, l'un des théoriciens les plus puissants de la sémiotique? Pertinence et pratique. Essai de sémiologie (1975) est le maitre-ouvrage de Prieto. Compact, l'ouvrage poursuit la réflexion des concepts issus de la linguistique structurale dans la double direction de leur extension à d'autres objets et d'une interrogation de type philosophique. Il y est question de la langue et des signes, mais également des sciences humaines et de l'idéologie. Autant que par l'ampleur de vues, l'ouvrage en impose par la maîtrise de son argumentation théorique. Et le style est d'une parfaite clarté. Néanmoins, là encore, au contraire de la théorie greimassienne, il faut convenir que la théorie sémiotique présentée dans Pertinence et pratique n'a guère fait école.

3 Si Luis J. Prieto fut tout cela - un des premiers sémioticiens et un grand théoricien -, il demeure dans nos mémoires tel un outsider magnifique. Combien de fois n'ai-je entendu cette phrase assassine, qui a l'air d'admettre par principe ce qu'elle dénie par le fait: - Prieto ? Ah oui, mais plus personne ne le lit.

4 Je voudrais tenter pour cet oubli un début d'explication. Il me parait que Prieto, par bien des côtés, n'est pas tant le contemporain de Greimas que celui de Hjelmslev. Et son œuvre relève des signes avant-coureurs de la sémiotique plutôt que de ses débuts effectifs. Le terme de sémiologie auquel est attachée cette œuvre en est un symptôme: Prieto faisait 
encore de la sémiologie quand la sémiotique avait entrepris de se constituer en tant que discipline.

Herman Parret, dans Language and Discourse, a bien décrit la singularité de cette position: l'héritage théorique de Saussure doit être partagé entre Hjelmslev et Prieto, et fonde par là même une opposition qui, bien qu'elle dénote un certain anachronisme, se justifie sur le plan des considérations théoriques (cf. Parret 1970). Car c'est bien de Hjelmslev que Prieto s'est fait, avec le plus de constance, le contradicteur. Du reste, Prieto partage avec le maître de Copenhague un même langage: un style "abstrait» et classique, un vocabulaire technique pris dans une syntaxe non formalisée, un intertexte de références qui comprend l'œuvre de Saussure, les travaux de phonologie structurale et les projets du logicisme. En revanche, comme ceux de Hjelmslev, les textes de Prieto sont à peu près dénués d'érudition philosophique explicite ${ }^{3}$. Et ils font peu allusion aux travaux d'autres sémioticiens ${ }^{4}$.

\section{Linguistique et sémiologie, aller-retour}

6 Plus globalement, ce qui apparaît ici est un certain rapport entre la linguistique et la sémiotique, qui rend peut-être compte également de l'ambivalence de la sémiotique / sémiologie. Prieto est de ceux-là, ayant traversé un océan, pour qui il n’y a qu'une rive - la linguistique - devant le large - la sémiologie.

De fait, Prieto a toujours fait ses réflexions sémiologiques depuis la linguistique, en particulier depuis la phonologie structurale ; c'est là que trouvent leur origine la plupart de ses exemples et de ses concepts théoriques. Et en son temps il a pris parti, résolument, pour une théorie linguistique particulière, celle défendue par André Martinet.

8 Son œuvre parcourt ainsi une double trajectoire : de la linguistique à la sémiologie ; de la sémiologie à la linguistique. À l'aller : la linguistique fournit une batterie de concepts et de conceptions que le sémiologue tire vers le général : généralisation des concepts et généralisation de leurs applications. La linguistique prend alors le rôle de science-pilote parmi les sciences sémiologiques à venir, c'est-à-dire parmi les sémiologies des systèmes particuliers que sont, par exemple, la musique, le cinéma, l'architecture, le récit. Au retour : cette généralité cherche à fournir à la linguistique une assise théorique d'un type différent de celle que les linguistes peuvent élaborer.

9 Et en ce sens, il est vrai, les travaux sémiologiques de Prieto se montrent fidèles au projet saussurien. La sémiologie accomplit d'abord un programme d'applications particulières mais, ce faisant, elle assure aussi à la linguistique un statut épistémologique qui, sans elle, lui ferait défaut.

10 Reste à déterminer quelle est la réflexion théorique offerte par la sémiologie de Prieto. Il est communément admis que les vues de Prieto consacre dans le milieu intellectuel français l'opposition entre Prague et Copenhague. Le point de vue adopté est en effet explicitement un point de vue "substantialiste» (1975: 88). Mais les guillemets qui entourent le qualificatif montrent qu'il ne va peut-être pas de soi ${ }^{5}$.

11 Les questions que je voudrais poser à l'endroit de l'œuvre de Prieto sont dès lors les suivantes: Que peut apporter la réflexion sémiologique à la linguistique? Qu'est-ce qui différencie celle-ci des propositions théoriques internes à la discipline linguistique? Comment, à quel niveau et par quels moyens, se règle un différend dans ce type de 
réflexion? Enfin, de tout cela, quel enseignement les sémioticiens contemporains peuvent-ils espérer?

Pour donner quelque substance à ces questions, il faut entrer dans les textes. Et pour ne pas avoir à répéter la monumentale analyse de Parret déjà citée, je propose d'examiner ici un des derniers articles de Prieto, consacré au problème de la syntaxe. Intitulé "Caractéristique et dimension. Essai de définition de la syntaxe », cet essai n'a pas encore fait, à ma connaissance, l'objet d'une discussion. Marie-ClaudeCapt-Artaud affirme toutefois qu'il représente "l'aboutissement de l'effort mené par les Saussuriens pour définir les traits pertinents » $(1994: 10)$. C'est là, comme on va le voir, un avis que je suis loin de partager. Je compte montrer que la théorie de Prieto, toute brillante qu'elle soit, manifeste au contraire une sorte de capitulation, par rapport aux préceptes épistémologiques de Saussure, devant la toute puissance de la théorie chomskyenne et de l'épistémologie logicienne à laquelle celle-ci est asservie.

13 Néanmoins, je prie le lecteur de ne pas perdre de vue que, menant ici une critique de la sémiologie selon Prieto, mon objectif est de faire ressortir les différents aspects argumentatifs qui dans cette pensée sont destinés à la linguistique.

\section{Dimension d'objet}

14 La théorie sémiologique de Prieto part d'une définition de l'objet en tant qu'unité. En tant qu'unité, un objet est pourvu d'une caractéristique, grâce à laquelle il diffère d'autres objets sur la base d'une dimension. La dimension, que Prieto appelle également élément contrastif ${ }^{5}$, est "un point de vue duquel on considère l'objet auquel on reconnaît la caractéristique en question» (1988: 30). Par exemple, l'objet /porte/ a une caractéristique d'avoir 《2,8 mètres de hauteur $»^{7}:$ la « hauteur » constitue la dimension sur laquelle se caractérise l'objet, en s'opposant aux autres objets par le fait d'avoir une hauteur particulière, «2,8 mètres ». Prieto appelle cette partie distinctive de la caractéristique l'élément oppositionnel.

Apprécions les prémisses épistémologiques d'une telle définition. D’abord, elle relève pleinement d'une épistémologie, et non d'une ontologie, puisque la description de la caractéristique constitue exclusivement la description de l'objet, et qu'il s'agit par conséquent d'un objet-pour la connaissance, dont on ne présuppose que l'appréhension phénoménologique. Ensuite, elle pose directement la question de l'unité de l'objet : est un l'objet qui est connu comme constant dans sa différence vis-à-vis des autres objets.

Encore appelle-t-elle une définition des possibilités de la connaissance, sans quoi le risque d'hypostase à un monde pré-donné resterait trop menaçant. Cette définition est celle de l' univers du discours :

[...] seules apparaissent à un sujet comme constituant des objets les portions de la réalité qu'il reconnait comme faisant partie d'un univers du discours. Une portion de la réalité étant donnée, qui apparaît à un sujet comme constituant un objet, les autres objets dont le premier diffère ou non sont les autres portions de la réalité que le sujet reconnaît comme faisant partie du même univers du discours, grâce à quoi elles lui apparaissent comme constituant elles aussi des objets (1988 : 26-27).

Dans cette définition, on le voit, rien n'induit la nécessité d'une connaissance qui soit homogène. Pas davantage, l'univers du discours n'est-il préexistant ou sa connaissance préalable à la connaissance des objets qu'il constitue. Au contraire, la possibilité y est laissée bien grande pour un savoir pluriel et dynamique, évitant autant que nécessaire 
une naturalisation chosiste des objets, quels qu'ils soient. Les caractéristiques sont donc tout à la fois intrinsèques à cet objet et inconsistantes.

Bien qu'il s'agisse là d'une définition de l'objet en général, Prieto vise aussitôt un objet particulier: le son langagier. Ainsi, le son [e], en français, sera constitué de deux caractéristiques - la position antérieure de la langue et la position non arrondie des lèvres - de façon à ce qu'il se distingue de $[\varepsilon]$ et de [o]. En revanche, en italien, comme $[\varepsilon]$ et [e] ne sont pas des objets, la position antérieure de la langue et la position non arrondie des lèvres ne constituent pas deux caractéristiques mais une seule, permettant de distinguer $[\mathrm{e}]$ de $[\mathrm{o}]$. Cette procédure d'analyse linguistique est très semblable à l'analyse promue par les phonologues pragois. L'univers de discours y joue le rôle de système ${ }^{8}$, les objets étant définis par des caractéristiques de la même façon que les phonèmes le sont par des traits pertinents.

L'inconsistance de l'objet ne l'empêche pas, dans un second temps, de prendre une identité . Pour Prieto, l'identité d'un objet est liée à une intentionnalité empirique: elle est constituée par la caractéristique de l'objet que le sujet considère comme pertinente. Et, fait-il remarquer, c'est «la dimension elle-même qui est pertinente pour le sujet logiquement avant que ne le soient des caractéristiques comportant cette dimension comme élément contrastif » $(1988: 35)^{9}$. Ainsi, l'identité d'un objet est-elle arrêtée par un état de pertinence qui le présentifie à l'égard d'un sujet en fonction prioritairement du point de vue contrastif, lequel est lui-même identifié. La caractéristique «2,8 mètres de hauteur » n'est pertinente que pour autant que d'autres portes soient déterminées par le même élément contrastif que constitue la « hauteur ». Sans doute la « hauteur » n'est-elle pas une caractéristique «en soi » de la porte; il a fallu néanmoins l'identifier afin que, dans un temps logiquement second, on puisse à partir d'elle comparer différentes portes et différentes hauteurs de portes.

En ce sens, le point de vue peut se " détacher », tout au moins analytiquement, de l'objet qu'il constitue. On retrouve alors l'acception ordinaire d'une classe entendue comme « ensemble » rassemblant des objets communs. La classe ne trouve d'extériorité vis-à-vis de l'objet à la constitution duquel elle participe qu'à condition de cette intention de pertinence qui l'affuble d'une identité positive. Mais cette extériorité et cette identité sont secondaires par rapport à l'espèce d'intériorité différentielle ou non consistante dans laquelle sont d'abord constitués l'objet et sa caractéristique.

\section{Dimension syntaxique d'objet}

21 Il existe toutefois d'après Prieto un type particulier de caractéristiques qui va permettre de spécifier l'objet linguistique parmi les objets en général. Dans ces caractéristiques "spéciales " « l'élément oppositionnel est constitué à son tour par un objet déterminé, c'est-à-dire un objet possédant une certaine identité et possédant donc à son tour certaines caractéristiques » (1988: 38). Deux sortes d'objets sont déterminées par ce type particulier de caractéristiques. La première sorte est attendue: il s'agit des objets composés; la seconde, en revanche, nous interpelle: sont syntaxiques, pour Prieto, les objets qu'on appelle les sens.

Toutes deux concernent de façon éloquente l'étude linguistique et permettent de dégager dans le contexte de cette étude une définition inédite pour le concept de "syntaxe ». Car, pour Prieto, l'élément contrastif d'une caractéristique dont l'élément oppositionnel 
constitue un objet correspond à ce que doit être nécessairement (et exclusivement, pour peu qu'on veuille bien étendre l'application du terme à toutes les possibilités ouvertes par le concept ainsi défini) une dimension syntaxique.

Commençons par présenter un exemple non linguistique d'objets composés. Soit un numéro de téléphone. C'est un objet composé dont les objets composants - les chiffres ont pour dimension syntaxique la position qu'ils occupent dans l'objet composé, et pour élément oppositionnel eux-mêmes, en tant qu'ils sont identifiés comme des objets.

Deux précisions définitionnelles limitent la portée du concept d'objet composé. Seule la première, toutefois, a été prise en compte par Prieto.

Premièrement, les objets composés sont toujours des objets matériels. On ne pourrait, sans cela, parvenir à identifier les objets composants, tels que les chiffres. Le numéro de téléphone identifié comme objet composé doit par conséquent être inscrit dans une matière - par exemple la matière lumineuse des chiffres digitaux - dans laquelle il est possible d'identifier, effectivement, les différents chiffres qui le composent. Pour ce faire, il faudra considérer que le chiffre digital est à son tour un objet composé, dont le nouvel objet composant a pour élément oppositionnel l'objet identifié comme étant un /bâton lumineux/ et pour nouvel élément contrastif les sept positions que peut occuper ce bâton lumineux.

Il apparaît que par le truchement de cette analyse des objets composés on ne puisse parvenir à identifier ce qu'est un numéro de téléphone « en général », c'est-à-dire tel qu'il peut se présenter à nous sans trouver à s'inscrire dans aucune matière particulière. Selon Prieto, cet objet général n'est pas un objet composé, mais bien une « idée » ou un « sens », c'est-à-dire un objet appartenant à la deuxième catégorie d'objets dotés d'une dimension syntaxique. C'est donc là, lors de la présentation de cette catégorie d'objets, que nous retrouverons le numéro de téléphone " général ».

La seconde précision à indiquer quand on fait l'analyse d'un objet composé est qu'il ne faut pas confondre l'objet composant avec son élément oppositionnel. L'objet composant d'un numéro de téléphone, ce n'est pas /tel chiffre/ - cela, c'est seulement son élément oppositionnel - mais plus exactement /tel chiffre dans telle position/. Mais dans ce cas, en opposition avec le premier point de précision, la qualification de matériel n'est pas tout à fait adéquate ; il aurait mieux valu dire que l'objet composé est, comme ses composants, phénoménal.

28 Voyons à présent une application dans le domaine de l'expression linguistique. On conviendra sans peine, sur le modèle du cas précédent, que le mot écrit est un objet composé des objets composants que sont les graphèmes. En français, ces graphèmes peuvent connaître jusqu'à 25 dimensions syntaxiques (d'après le nombre de lettres du mot ordinairement admis pour être le plus long de la langue française). Les dimensions syntaxiques $d u$ graphème correspondent aux différentes positions qu'il peut occuper dans le mot écrit. Ces dimensions syntaxiques, et les régularités qu'elles observent, constitue ce qu'on pourrait appeler, avec Prieto, une syntaxe graphique.

Plus intéressante est l'application à l'expression phonolinguistique. Une syntaxe phonologique, d'après Prieto, doit partir de la syllabe, unité première de cette phonosyntaxe (tout au moins concernant la langue française), retrouvant par là la théorie phonologique de Saussure, en accord également avec les développements cénématiques de la glossématique. Cette syllabe est l'objet composant d'une cadence; son élément contrastif désigne /telle position/ dans la cadence; elle-même constitue un objet 
composé, dont les objets composants ont pour élément contrastif /telle position (prévocalique, vocalique ou postvocalique)/ dans la syllabe. Le son est l'élément oppositionnel de cet objet composant, mais lui-même, on l'a vu, n'est pas un objet composé. La stratification des analyses d'identités syntaxiques finit donc par s'arrêter à des objets minimaux qui n'appartiennent pas à la catégorie particulière des objets composés.

\section{Dimension syntaxique du sens}

Voyons à présent la seconde catégorie particulière d'objets dotés d'une dimension syntaxique. Il s'agit, comme annoncé, de la catégorie du sens. Ou plutôt des sens, puisque pour Prieto, il n'y a que des sens identifiés, des sens qui sont « une connaissance de l'émetteur que celui-ci essaie de transmettre ou de communiquer au récepteur, c'est-à-dire de faire devenir aussi une connaissance de celui-ci » (1988: 47). Il faut donc au sens une pertinence attribuée par un sujet empirique. Cette pertinence est fonctionnelle : le sens a une fonction de communication. En dehors de cette fonction, pas de sens possible, pour Prieto, mais seulement des connaissances.

Or, en cette fonction, l'émetteur et le récepteur reconnaissent au sens deux identités. L'une relève de l'univers du discours de la compréhension; l'autre, de l'univers du discours linguistique. Par exemple, si l'on désire communiquer qu'on "préfère le vin rouge ", la référence au vin est une caractéristique du sens communiqué qui est pertinente au niveau de la compréhension. «Ensuite, » poursuit Prieto,

selon que l'émetteur, pour transmettre le sens, produit, par exemple, la phonie [39 pRefॄR l९ vẽ Ru3] (Je préfêre le vin rouge) - qui indique la présence dans le sens de la

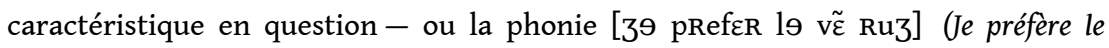
rouge) - qui ne l'indique pas -, cette caractéristique devient ou non pertinente aussi au niveau linguistique. (1988: 48 ; j'ai transcrit les énoncés selon l'alphabet phonétique international, et non comme dans l'original.)

L'identité du sens pertinente au niveau linguistique est le signifié, le signifiant n'étant rien d'autre, écrit Prieto, « que l'identité linguistique de la phonie » (ibidem).

La dimension syntaxique apparaît dès lors comme suit : dans la phonie /Il a perdu votre numéro de téléphone/, on identifie notamment un objet dont l'élément oppositionnel est "le numéro de téléphone de l'interlocuteur " (sans précision d'une quelconque matière qui rendrait sensible ce numéro de téléphone) et l'élément contrastif la dimension " (objet direct)». L'élément oppositionnel correspond au référent, qui appartient à l'univers de compréhension du locuteur, tandis que l'élément contrastif, exprime une dimension syntaxique affirmant l'univers linguistique du même locuteur.

Voici donc qu'avec Prieto la seule préoccupation qui soit assignée à la linguistique, c'est la syntaxe (à laquelle, certes, est annexée une syntaxe phonologique). En, revanche, la notion de paradigme linguistique est totalement évacuée. L'analyse paradigmatique relève pour lui d'un autre univers de discours, concomitant dans la phonie à l'univers linguistique : à savoir celui identifié par les locuteurs comme l'univers des référents.

Qui s'inquiéterait de voir ainsi l'univers linguistique assujetti à un univers extralinguistique peut se rassurer. Prieto prend soin en effet d'ajouter que la langue joue un rôle déterminant dans la constitution des identités du sens pertinentes au niveau de la compréhension. 
La «forme» du sens au niveau de la compréhension est donc éminemment linguistique, même si, une fois devenue instrument de la pensée (on pense « avec sa langue ", ce qui veut dire avant tout, à mon avis, que nos contenus mentaux sont déterminés par des caractéristiques dont les éléments contrastifs sont ceux qui ont été imposés par la langue), elle n'est pas toujours entièrement (re)rendue pertinente au niveau linguistique lorsqu'il s'agit de communiquer cette pensée.

(1988: 50.) partie du sens transmis par la phonie bouleversent considérablement la tradition de la sémantique lexicologique. Ainsi, pour Prieto, les verbes n'ont pas de dimension syntaxique. Ils ne sauraient par conséquent avoir à proprement parler de sens (puisque le sens est défini par le fait d'avoir une dimension syntaxique). De fait, l'élément oppositionnel des verbes ne constitue pas, dans l'univers de compréhension, un objet. Tout au plus participent-ils au sens, sans trouver à s'en distinguer (et donc sans dégager de forme qui puisse valoir généralement au niveau linguistique). À l'inverse, les pronoms renvoient à une identité au niveau de la compréhension qui n'est pas reprise au niveau linguistique. Leur analyse ne saurait donc se limiter au seul niveau linguistique. Ainsi, dans /Il a perdu votre numéro de téléphone/, le pronom renverrait nécessairement, au niveau de la compréhension, à un quidam particulier mais, au niveau linguistique, seule serait pertinente la caractéristique "(sujet) il». (Mais qu'arrive-t-il à ce niveau de compréhension, si, comme dans le cas présent, la phonie sert simplement d'exemple dans une étude théorique et n'entend pas le moins du monde référer le /il/ à quelque quidam que ce soit en particulier?) Quant à "(verbe) perdre», il présenterait un élément oppositionnel, «perdre », qui n'est pas un référent, et n'est donc nullement pertinent au niveau de la compréhension. (Mais comment passe-t-on alors de /a perdu/ à « perdre »? Comment identifie-t-on une connaissance qui ne fait pas sens ?)

craindre que dans ces derniers aspects, l'analyse impliquée par cette conception très originale de la syntaxe, et la délégation du sens qu'elle permet à un univers non linguistique, posent autant de problèmes qu'elles n'en résolvent. Laissons-lui néanmoins le bénéfice d'un perfectionnement virtuel.

\section{Identité et unité}

Je voudrais à présent approfondir quelques-uns des points théoriques impliqués dans la définition générale, sémiologique, de la syntaxe. En guise de contrepoints, je me servirai de certains arguments sémiotiques de Hjelmslev afin de montrer, par comparaison, que les options théoriques de Prieto s'avèrent plus problématiques qu'elles n'y paraissent à première vue.

40 Tout d'abord, on peut se demander sur quelle base exactement se définit un univers de discours. Que cet univers soit nécessaire, cela ne fait pas de doute; il constitue un 
préalable à toute connaissance, puisqu'il permet de rassembler ce qui est irrémédiablement particulier dans un geste, un postulat, de généralisation.

Hjelmslev, semblablement, a été tenu d'indiquer par le terme de texte le fait dont l'analyse linguistique tâche de décrire la spécificité - entendue: la spécificité générique, la spécificité permettant précisément de l'appréhender sous le concept général de texte. Mais Hjelmslev a pris soin de bien distinguer le texte de l'objet décrit par la linguistique, à savoir les formes et le système linguistiques; faute de quoi il aurait été bien en peine d'expliquer comment cette généralisation, qui, comme toute généralisation, est une généralisation relative, peut atteindre la spécificité du fait linguistique. Car, de deux choses l'une : ou la spécificité du texte est établie préalablement à l'analyse, et alors on est en droit de se demander à quoi celle-ci peut encore servir; ou la généralisation relative qui atteint cette spécificité n'est reconnue qu'une fois l'analyse réalisée, et on ne peut dès lors poser a priori que le texte, ou l'univers du discours, comme on voudra bien le nommer, est le dépositaire de la spécificité que lui assigne sa connaissance.

De ce petit syllogisme, il doit ressortir une chose bien claire : il n'est pas licite de fonder une analyse sur la distinction, identifiée comme pertinente préalablement à cette analyse, de deux univers de discours. Et, pour le dire avec une précision théoriquement superflue, mais qui dissipera peut-être tout malentendu : ce n'est pas que deux univers de discours ne puissent être pertinents ; bien au contraire, il faut saluer l'ouverture épistémologique apportée par Prieto, avec ce concept d'univers du discours, vers une connaissance plurielle, hétérogène et dynamique. Ainsi, pour reprendre un exemple familier à JeanMarie Klinkenberg ${ }^{10}$, un trait de fumée est-il certainement pertinent dans l'univers du discours pictural quand il s'agit de dessiner un bateau à vapeur; il ne l'est pas dans l'univers de description scientifique de ce bateau. Plusieurs univers de discours peuvent donc coexister dans le chef d'un même individu, qu'ils y apportent ou non le germe de la contradiction. Mais ils ne pourront jamais être pertinents en même temps.

C'est cela, néanmoins, qu'entend proposer Prieto quand il aborde le sens. Le sens est pour lui un objet auquel on reconnaitrait en même temps deux identités. C'est à mon avis indéfendable; car l'identité intentée à un objet est dépendante de la phénoménalité de cet objet, toujours absolument particulière; aussi cette identité ne peut-elle pas se dédoubler sans remettre en cause l'idée même de phénomène.

On en vient alors à douter que soient suivies par Prieto les prémisses épistémologiques qu'il a pourtant explicitées et qui ont si bon air. Par exemple, dans le commentaire sur la pertinence, lit-on sous sa plume: «[...] la pertinence d'une caractéristique d'un objet suppose la pertinence dans au moins un des objets dont il diffère par la caractéristique en question de la caractéristique comportant le même élément contrastif que ce dernier objet présente nécessairement " (1988: 34 ; je souligne). De quelle forme de "présence » s'agit-il ? Il y a dans ce mot présence au moins un choix malheureux, encore aggravé par la condition exprimée par dans au moins un des objets, car il entre en contradiction avec la définition de la caractéristique. Est-ce à dire, en effet, qu'un élément contrastif saurait être "présent » tout en n'étant pas pertinent? Ce serait permettre la réintroduction d'une ontologie que la définition de la caractéristique avait permis d'éviter : une présence qui n'est pas reconnue par le sujet connaissant sort nécessairement du cadre tracé par l'épistémologie.

Or la séparation qu'autorise le concept de présence entre l'objet et sa caractéristique est encore beaucoup plus embarrassante dans l'autre sens; qui plus est, ce n'est pas seulement un risque qu'elle suscite alors, mais une contradiction explicitement soutenue 
par Prieto. Selon lui, en effet, il serait possible qu'une caractéristique soit pertinente tout en n'étant pas présente dans l'objet ! C'est ce que donne à lire en tout cas un passage déjà

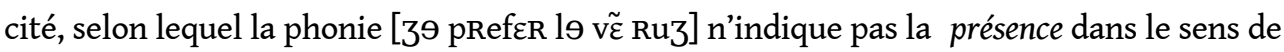
la caractéristique que constitue la référence au vin, laquelle est pertinente au niveau de la compréhension $^{11}$ (cf. 1988: 48). L'objet qui présenterait une telle caractéristique serait non phénoménal et découvrirait une intentionnalité constituante. Comment, dans ces conditions, pourrait-on encore maintenir que le sens est une connaissance communiquée ? À moins de prévoir un univers de discours préalablement constitué à la communication linguistique et en fonction duquel une caractéristique pourrait être transmise jusqu'en son absence - ce qu'infirme la définition de l'univers de discours -, une telle possibilité n'existe plus.

On ne saurait donc constituer un objet - fût-il le sens transmis dans la communication linguistique - en fonction de deux identités appartenant chacune à un univers de discours distinct. Encore moins pourra-t-on mettre l'un de ces univers - celui que Prieto prend comme spécifiquement linguistique - sous la dépendance de l'autre.

\section{Identité et différence}

Le point qui est discuté ici emporte avec lui la cohérence de l'appareil théorique de Prieto tout entier. Ce sont, en effet, dans les fondements de cette théorie, qui sont restés à peu près inchangés depuis les premiers essais de l'auteur, que se situe le problème de la caractéristique et de sa "présence » dans l'objet. Abandonnons un instant l'examen de l'article consacré à la définition de la syntaxe pour revenir sur un autre essai, imposant à tous égards, où Prieto a longuement développé sa conception de la caractéristique ; il s'agit, dans Pertinence et pratique, du chapitre intitulé « Langue et parole ».

Pour Prieto, la caractéristique est le corrélat nécessaire de la différence :

On ne reconnaît pas en effet un objet comme étant différent d'un autre objet sans lui reconnaître une caractéristique qu'il comporte et par laquelle il diffère de cet autre objet, et on ne reconnaît pas à un objet une caractéristique qu'il comporte sans reconnaître qu'il diffère, par cette caractéristique, d'un autre objet. $(1975: 82$.)

Cette proposition serait irréprochable si elle n'entraînait Prieto à inférer directement de la différence la définition de la classe logique :

L'identité qu'un sujet reconnaît à un objet est déterminée par la classe à travers laquelle il le connaît, c'est-à-dire par les objets dont il le reconnaît comme étant différent et par les caractéristiques qu'il lui reconnaît en conséquence. Deux objets apparaissent à un sujet comme étant identiques entre eux lorsqu'il leur reconnaît la même identité, c'est-à-dire lorsqu'il les connaît à travers une même classe et qu'il les reconnaît donc comme étant respectivement différents des mêmes autres objets. (1975 : 83.)

Que deux objets soient différents d'un troisième, il ne s'ensuit pas nécessairement, contrairement à ce qu'infère Prieto dans cette citation, qu'ils aient la "même" caractéristique, telle que la définit la citation précédente. La question de l'identité de la caractéristique doit être distinguée de la corrélation entre caractéristique et différence. Prieto remarque d'ailleurs, mais sans en tirer parti, que

reconnaître une différence entre deux objets, ce n'est rien d'autre que de reconnaitre l'un comme membre d'une classe et l'autre comme membre du complément correspondant. Or cela montre encore une fois la faiblesse de la notion traditionnelle d'« extension » d'une classe. Une classe apparaît en effet comme 
" unimembre» ou comme "plurimembre» et, dans ce dernier cas, comme différemment "plurimembre ", selon les classes avec lesquelles on est capable d'opérer. (1975: 84.)

51 Une " classe " dans son acception logique est toujours une classe homogène. On y traite toujours de la même manière les différences entre membres appartenant à une même classe et les différences entre membres appartenant à des classes distinctes. Or la corrélation entre caractéristique et différence ne découle que du seul type de rapport de différences entre membres de différentes classes; de sorte que la classe unimembre que pourrait définir le rapport de différence entre membres de cette classe n'implique aucunement la possibilité de la classe plurimembre.

Il s'ensuit deux corollaires. Premièrement, l'identité que l'on reconnaît aux membres d'une classe plurimembre est nécessairement secondaire par rapport aux différences qui constituent cette classe comme plurimembre. Deuxièmement, il ne saurait y avoir de caractéristique universelle sinon purement axiomatique visant à déterminer une classe plurimembre comme univers de discours.

53 C'est pourtant cette caractéristique universelle qui étaie la thèse «substantialiste » de Prieto:

Le schéma :

$u a \neq u \tilde{a}$

par lequel on peut représenter le calcul que suppose le fait de reconnaître une caractéristique qu'un objet comporte, est aussi, par conséquent, le schéma selon lequel s'établit toute différence; autrement dit, une différence s'établit toujours, certes, grâce à une présence $(a)$ et à une absence $(\tilde{a})$, mais entre deux objets qui apparaissent comme tels grâce à une présence $(u) .(1975: 90$.

54 Cette caractéristique universelle n'est pas autre chose, concernant l'opposition expression vs contenu, qu'une détermination ontologique. Pour Prieto, un phonème est distinct d'un autre phonème à condition qu'ils soient tous deux d'abord déterminés comme des phonèmes possibles en ce qu'ils participent de la substance phonique. La caractéristique universelle circonscrit le champ de l'analyse linguistique. Cette analyse a alors une immanence relative : relative à un champ déterminé ontologiquement. La négativité des formes y est également relative : c'est la négativité de formes dans un champ d'abord défini par une caractéristique positive. On pourrait maintenir encore que l'analyse linguistique est immanente, et négatives les formes de cette analyse, à condition de reconnaître qu'il s'agisse d'une analyse régionale. Cette analyse n'a pas alors à se munir d'une épistémologie qui lui soit propre, car la spécificité de son objet est déterminée en dehors d'elle.

Mais, surtout, l'acceptation d'une caractéristique universelle déterminant dans la substance l'expression et le contenu implique une analyse formelle conduite en terme d'invariabilité. Si la substance est déterminée, l'unité précède nécessairement les relations entre unités, et c'est alors dans un sens tout à fait impropre, qui confond substance et système, que l'on affirme que le système précède les unités du système. Et si l'unité précède les relations qu'elle connaît avec d'autres unités dans le système, sa formalisation ne peut plus admettre qu'elle varie, puisque c'est sa « stabilité » qui permet d'établir ses relations (syntagmatiques comme paradigmatiques) avec les autres unités du système. Certes, l'identification de l'unité "dépend » encore dans cette perspective de l'identité attribuée aux autres unités, mais seulement en fonction d'une identité préalablement attribuée par «corrélation » avec les unités de l'autre plan. Dans cette 
optique, l'antécédance n'est plus vraiment fixée entre système et unité, mais entre système et corrélation d'une unité d'expression avec une unité de contenu, c'est-à-dire que l'opposition expression vs contenu est devenue une détermination ontologique.

Ici se découvre le véritable enjeu de la théorie de la pertinence : qui n'est pas seulement de reconnaitre qu'il n'y a d'analyse de l'expression qu'en fonction d'une analyse de contenu, mais qui entend également imposer que le contenu et l'expression existent l'un et l'autre de façon distincte avant la formalisation linguistique que permet leur analyse.

Prieto accorde ici un crédit illimité à une image du Cours de linguistique générale selon laquelle les formes linguistiques sont prises entre deux zones amorphes qui ont l'air de préexister à leur formalisation, le plan indéfini des idées confuses et celui non moins indéterminé des sons (cf. Saussure 1916 : 162). Il est alors extrêmement regrettable que les rédacteurs n'aient pas suivi ici à la lettre les notes des étudiants. Dans le CLG, l'image est précédée de cet avertissement : « La substance phonique n'est pas plus fixe ni plus rigide ; ce n'est pas un moule dont la pensée doive nécessairement épouser les formes ". (Saussure 1916:161). Les notes de Riedlinger du deuxième cours ne concordent pas tout à fait avec cet avertissement : « Mais il ne faut pas tomber dans l'idée banale que le langage est un moule : c'est le considérer comme quelque chose de fixe, de rigide, alors que la matière phonique est aussi chaotique en soi que la pensée. » (Saussure 1968, 1.252.1826.2). Il est clair, selon ce texte, non seulement que la substance phonique n'est pas un moule mais que le langage n'en est pas un non plus; qu'il n'a rien de fixe. Juste après, Riedlinger ajoute d'ailleurs, sous la dictée de Saussure - le professeur Saussure, non le narrateur du CLG où les propos qui suivent sont également déformés :

Ce n'est pas la matérialisation de ces pensées par un son qui est un phénomène utile ; c'est le fait en quelque sorte mystérieux que la pensée-son implique des divisions qui sont les unités finales de la linguistique. Son et pensée ne peuvent se combiner que par ces unités. (Saussure 1968, 1.253.1830.2)

Est-il un seul passage du CLG où soit fixée de manière aussi explicite l'antécédance des relations ou divisions - qui sont les unités finales de la linguistique - sur les unités que constituent la combinaison d'un son et d'une pensée! De fait, lorsqu'on se résigne à faire de l'opposition expression vs contenu une détermination ontologique, c'est-à-dire quand on croit pouvoir en faire une pensée et une masse sonore qui existeraient en soi comme des sortes de nébuleuses indifférenciées, on se résigne également à faire de la langue un facteur de stabilité entre ces deux nébuleuses. On cantonne a priori la langue aux limites de la représentation que l'on peut s'en faire. Or la langue est tout le contraire : c'est une activité, une énergétique, un événement; un ordre, certes, et une hiérarchie, mais un ordre et une hiérarchie dynamiques, incessamment variables, constamment remis en cause. Et ce n'est que la représentation qui saisit (comme on dit tout à la fois du gel qu'il saisit la nature et de l'esprit qu'il saisit un raisonnement) la variabilité invariable de la langue en une coupe invariable de variétés.

\section{Une syntaxe universelle?}

60 On peut revenir alors à la critique de "Caractéristique et dimension ». Les effets d'une analyse résignée à l'invariabilité formelle, telle qu'elle est impliquée par la détermination ontologique de l'expression et du contenu, apparait nettement dans la présentation théorique que fait Prieto de la syntaxe. Quand, chez Hjelmslev, la syntagmatique est théoriquement antérieure à la paradigmatique et que leurs actions analytiques sur le fait 
linguistique se contiennent mutuellement, chez Prieto, au contraire, la syntaxe vient nécessairement après le classement paradigmatique ou, plus exactement, après ce qui en tient lieu, à savoir l'identité du sens pertinente au niveau de la compréhension (que plus loin l'auteur désigne également sous le terme de sémantique, retrouvant ainsi la conception logicienne de l'opposition syntaxe $v s$ sémantique).

61 Souvenons-nous, en effet, que le sens d'après Prieto est un objet possédant une dimension syntaxique parce que son élément oppositionnel est lui-même un objet. C'est le "référent", pertinent au niveau de la compréhension, qui est cet objet tandis que l'identité pertinente au niveau linguistique forme la dimension syntaxique du sens.

L'identité du sens pertinente au niveau linguistique, c'est-à-dire le signifié du signal employé pour le transmettre est donc une identité que l'on reconnaît à un objet - le sens - déjà déterminé par une autre identité logiquement antérieure - l'identité pertinente au niveau de la compréhension. (1988: 49.)

Mais, paradoxalement, bien que la syntaxe soit dépendante de la sémantique, elle développe également la partie universelle du sens :

[...] la distinction qui permet le mieux de délimiter le domaine de la syntaxe n'est pas à mon avis la distinction entre «forme » et « substance » mais la distinction, à l'intérieur même de la substance, entre ce qui, dans la construction cognitive que l'on en fait, apparaît comme étant universel et ce qui, dans cette même construction, apparaît comme ne l'étant pas et comme étant par conséquent distinctif.

[...]

À l'encontre de la syntaxe, qui étudierait ce qui est universel dans la construction cognitive du sens, la sémantique étudierait ce qui y est distinctif. (1988: 62.)

À première vue, on serait porté à penser que Prieto donne raison ici à un sentiment ordinaire à propos du langage. La sémantique y fait le partage entre les mots, chacun distinct des autres, alors que la syntaxe considère que ces mots, rassemblés en grandes catégories, se valent quant à leur fonction dans la phrase. Selon ce sentiment ordinaire, il y a moins de sens dans "verbe " que dans chaque verbe particulier; si "verbe" appartient à la syntaxe (à son «métadiscours»), chaque verbe particulier, en revanche, est redevable d'une analyse sémantique qui le distingue des autres et lui permet d'être identifié sur le plan de l'« indication » phonique.

À y regarder de plus près, on se rend compte que cette interprétation n'est pas rejetée tout à fait par Prieto, mais que celui-ci la récupère au bénéfice d'un rapprochement avec la syntaxe "à arbres" de Chomsky. Mais, dans ce rapprochement avec la grammaire universelle, on peut pressentir que la spécificité des langues n'est pas tenue pour très importante et qu'elle cède le pas sur l'universalité de la pensée. Contrairement à ce que laissait accroire un sentiment premier, ce n'est donc pas une syntaxe et une sémantique linguistiques qui sont ici visées, mais bien une syntaxe et une sémantique de la " construction cognitive».

Et, de fait, en s'efforçant de s'y retrouver tout à fait, que constate-t-on? Premièrement, que la sémantique ne répond pas de la pertinence linguistique mais de la pertinence des référents. Deuxièmement, que la syntaxe, " pôle » linguistique de l'identification du sens, est entièrement sous la dépendance de la sémantique; on ne voit pas dès lors comment elle pourrait rendre compte de la diversité des langues, si ce n'est par une « re- »particularisation. Troisièmement, que la syntaxe a néanmoins vocation d'universalité ; en conséquence de quoi, l'ouverture envisagée au second point est rendue caduque et l'on ne voit plus du tout dès lors en quoi la syntaxe est linguistique, si ce n'est en fin de compte pour la simple raison qu'elle est liée à la phonie, c'est-à-dire à la 
«matière » nécessaire pour transmettre le sens. Prieto a beau jeu alors de reconnaître que l'univers de compréhension est informé par la langue. Dans la pratique de son analyse, cet univers de compréhension est bien coupé de sa constitution linguistique originelle et constitue un préalable à l'analyse linguistique.

Or, à tout le moins, ce préalable n'est-il pas plus aisément descriptible dans l'univers de compréhension que lorsqu'on en vise le contenu par l'analyse lexicologique traditionnelle. En fonction de quels critères, en effet, se constitue l'identité du sens pertinente au niveau de la compréhension? Le référent dépendra toujours du contexte d'énonciation de la phonie qui le signale. Comment dans ce cas peut-il conserver une

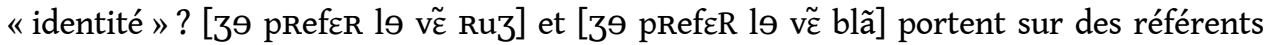
différents. Comment, dans l'analyse proposée par Prieto, l'identité du vin est-elle reconnue?

Il y a chez Prieto un déni de formalisation qui n'est pas tenable et qui remonte des profondeurs sous l'aspect d'une évidence naturelle. L'analyse par la pertinence, bien loin de dégager les identités du sens, ne fait le plus souvent que les confirmer en fonction d'une formalisation non reconnue mais néanmoins effective. Cela est vrai $a$ fortiori pour la dimension syntaxique. Si l'identité du sens au niveau linguistique dépend du référent, elle ne peut être stabilisée qu'en fonction d'une formalisation déjà accomplie avant l'analyse

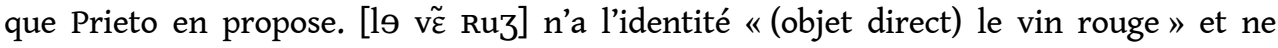

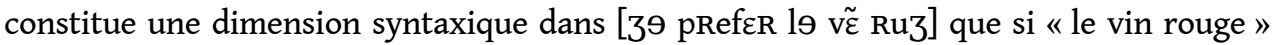
constitue une forme identifiée, que ce soit en fonction de l'univers de compréhension ou que ce soit en fonction d'une analyse paradigmatique (linguistique). On voit bien que c'est parce qu'est ignorée, tout au moins du point de vue de l'analyse linguistique, la

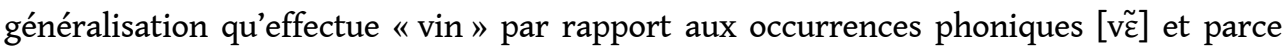
qu'au contraire ce général qu'est le sens identifié en fonction des référents est donné comme un particulier d'ores et déjà « distinctif », que la syntaxe a pu apparaitre comme la part d'universel dans l'identité du sens.

68 En réalité, s'il est si facile d'ignorer la nécessité, concomitante à l'analyse lexicologique, de la formalisation syntagmatique, c'est seulement parce que le langage, dans son analyse lexicologique, est susceptible de présenter une forme invariable autonymique qui a la même «substance " que les manifestations variables d'une forme linguistique. Par exemple, vin, autonyme de /vin/ et "vin», est ainsi confondu avec ces formes linguistiques. L'identification des référents dans l'univers de compréhension n'est évidemment pas autre chose que cette identification des autonymes, tels qu'ils pourraient apparaître comme "dégagés » des faits linguistiques, en lieu et place du sens que l'analyse lexicologique permet de formaliser.

Il se confirme ainsi que ce que vise Prieto, c'est une grammaire de la pensée, et non une grammaire linguistique.

Du reste, Hermann Parret a bien montré comment, des propositions équivoques du CLG, la pente était facile vers un sémantisme pur. Ce sémantisme, à la recherche d'un signifié transcendantal, va de pair avec une téléologie du langage (qui, pour le moins, est également une ontologie): la langue y est considérée comme transmission de significations. Le «fait mystérieux de la pensée-son» sur lequel méditait Saussure est devenu un fait transparent de sons transmettant "arbitrairement» du sens déjà constitué. La formalisation consiste alors à établir des invariables, de façon à maintenir ce 
sens préexistant en dépit de l'arbitraire linguistique, responsable des variations (dites syntaxiques) du sens spécifique à chaque langue.

Il est, de plus, facile de voir que phonologisme et sémantisme vont de pair [...] : ils isolent la factualité de la langue du contexte argumentatoire global chez Saussure, où le signe n'est pas seulement la relation sémiologique du signifiant et du signifié, mais en même temps le terme du système linguistique et l'unité de l'ordre syntagmatico-discursif. (Parret 1987 : 28-29.)

On ne s'étonnera plus dès lors que la sémiologie de Prieto se soit rapprochée de la théorie fonctionnaliste de Martinet, toutes deux issues de l'orientation pragoise de la linguistique structurale. En effet, quand une détermination ontologique « sépare » la pensée et le son préalablement à l'analyse linguistique, selon que le domaine de recherche est strictement limité à la linguistique ou bien qu'il s'étende vers la sémiologie, la théorie linguistique va pencher soit du côté de l'analyse phonologique et d'une analyse en "monèmes » de langues particulières, soit du côté d'une analyse universelle qui permette de comprendre comment se transmet le sens corrélé à "l'identité linguistique de la phonie » (le signifiant). Il suffit de désavouer la détermination ontologique de l'opposition pensée vs son, variété du dualisme platonicien, pour refuser le partenariat du sémantisme et du phonologisme.

\section{En conclusion}

Je crains que le lecteur aura trouvé cette suite théorique bien aride, et peu éclairante sur les questions que j'ai soulevées au début de mon article. Il est vrai que, dès qu'on s'aventure dans une argumentation de type sémiotique, la quantité d'abstraction qui y est requise risque à tout moment de faire perdre pied. On aurait sans doute souhaité que les exemples fassent voir un peu plus clairement ce dont il retourne, ou bien qu'apparaissent des formalisations de type logique auxquelles on puisse se raccrocher, ou bien encore que les concepts philosophiques évoqués le soient à partir d'un système de référence plus notoire. Mais justement, ce sont là des outils qui font ordinairement défaut aux sémioticiens. L'argumentation sémiotique a son langage propre, auquel il faut s'accoutumer. Quant aux exemples, ils sont rarement satisfaisants, dès lors que la sémiotique envisage une généralisation sur des exemples tirés d'une discipline où ils ont déjà, et souvent bien mieux, leur pertinence. Ainsi, ce qui permet davantage de " voir " un argument de théorie sémiotique, ce sont souvent, hélas, les contre-exemples qu'on avance à son encontre.

$\mathrm{Au}$ reste, je ne prétends pas que cette présentation critique permette de faire l'économie d'une lecture des textes de Prieto. Je souhaite au contraire que le lecteur qui ne s'y sera pas tout à fait retrouvé soit soucieux de retourner aux textes mis ici en examen et qu'il juge du bien-fondé de mes observations. Car je reste convaincu qu'il pourra y trouver son profit: la lecture de l'œuvre de Prieto reste d'une grande force de suggestion pour un grand nombre de questions philosophiques qui se rapportent aux sciences humaines, quand bien même on ne serait pas d'accord avec certaines de ses conclusions.

Néanmoins, il me semble que les critiques avancées lors de l'examen entrepris ici permettent de préciser quelques-uns des rapports qu'ont entretenus la sémiotique / sémiologie et la linguistique.

En développant sa sémiologie, Prieto a cherché à étendre certains concepts - tels le trait pertinent (ou caractéristique), la classe (ou dimension) et la syntaxe - issus d'une théorie 
linguistique pour les appliquer à d'autres phénomènes que les faits de langage verbal. Il a pu montrer ainsi leur puissance, mais il n'a pas toujours convaincu de leur adéquation. En effet, nombre des applications sémiologiques qui ont eu lieu dans les années soixante sont assez rudimentaires, et pour ainsi dire évidentes, en comparaison de l'analyse des faits de langage. Tel est le cas de l'analyse sémiologique du code de la route, des sémaphores, de la numérotation des chambres dans un hôtel ${ }^{12}$ ou encore des chiffres d'un cadran digital comme il en est question dans l'article examiné. La généralisation des concepts linguistiques n'est donc pas directement profitable à l'analyse de faits non linguistiques.

En réalité, l'élargissement sémiologique des concepts linguistique n'est pas requis par des besoins d'analyse mais bien plus sûrement en raison de considérations théoriques relatives à ces concepts. On constate d'ailleurs qu'aussitôt après avoir donné quelques exemples non linguistiques qui assurent la validité des généralisations conceptuelles ainsi visées, Prieto revient assez vite à des exemples linguistiques. L'exemple non linguistique n'aura été finalement qu'une sorte de détour, de prétexte, pour atteindre quelque considération particulière dans le domaine de la linguistique.

De quelle manière alors la sémiologie entend-elle redoubler la réflexion linguistique ? Par un effet d'extension, assurément, mais non pas tant relatif aux objets qu'au registre spéculatif de la connaissance. Ce à quoi prétend le sémiologue, c'est à inscrire les propositions théoriques qu'il explicite à propos de l'analyse linguistique dans un champ épistémologique plus large, celui des sciences humaines, sinon celui de l'ensemble du savoir. En retour, une telle ouverture épistémologique permet d'examiner les concepts linguistiques sous un angle inhabituel au sein du système théorique qui les a engendrés. Tel est le cas, par exemple, du concept de syntaxe qui, dans la visée sémiologique de Prieto, se voit articulé de façon singulière aux concepts de sens et de phonie.

Les arguments sémiologiques ont des caractéristiques formelles, un vocabulaire, des stratégies d'exposition qui leur sont propres et ils peuvent dès lors acquérir une certaine autonomie vis-à-vis des discours linguistiques ${ }^{13}$. Et la critique qui d'aventure s'exerce à leur endroit emploie des chemins étrangers aux routines analytiques des linguistes. Ces critiques, qui ont ici mis en avant un différend global "entre Prague et Copenhague ", sont développées sur le principe de la nécessité d'une cohérence épistémologique et en fonction des répercussions philosophiques de tels arguments. En l'occurrence, se réitère en sémiologie le débat infini entre positivistes et sceptiques, universalistes et relativistes.

Apparue avec le structuralisme, la sémiologie a eu un projet philosophique : elle a cherché à raisonner et situer la place des sciences du langage, et plus généralement celle des sciences humaines, dans une théorie de la connaissance renouvelée. La sémiotique peut-elle reprendre à son compte et actualiser un tel projet? Je ne pense pas qu'elle puisse le faire en tant que discipline constituée, et par des objets dont elle se réserverait l'étude - tout légitime que soit par ailleurs son programme. Néanmoins, par son histoire et par son approche du sens, elle est susceptible de contribuer à une réflexion d'épistémologie générale où les présupposés logiques céderaient enfin le pas devant les contraintes du langage naturel et les modalités culturelles de l'action sémiotique et herméneutique. 


\section{BIBLIOGRAPHIE}

CAPT-ARTAUD M-Cl. 1994 : Petit traité de rhétorique saussurienne, Genève, Droz.

HJELMSLEV L. 1943 : Prolégomènes à une théorie du langage, Paris, Minuit, 1968-71.

KLINKENBERG J-M. 1996 : Précis de sémiotique générale, Bruxelles, De Boeck Université, = Culture et communication.

PARRET H. 1971: Language and discourse, The Hague, Paris, Mouton.

- 1987 : Prolégomènes à la théorie de l'énonciation : de Husserl à la pragmatique, Berne, Peter

Lang, = Sciences pour la communication.

PRIETO L. J. 1964 : Principes de noölogie, The Hague, Paris, Mouton.

- 1966 : Messages et signaux, Paris, P.U.F., = Le linguiste.

- 1968 : « La sémiologie », Le langage, Paris, Gallimard, = La Pléiade, pp. 93-144.

- 1975 : Pertinence et pratique. Essai de sémiologie, Paris, Minuit, = Le sens commun.

- 1975b : Études de linguistique et de sémiologie générales, Genève, Droz.

- 1988 : «Caractéristique et dimension. Essai de définition de la syntaxe », Cahiers Ferdinand de Saussure, 42, pp. 25-63.

-1990 : "Classe et concept. Sur la pertinence et sur les rapports saussuriens "de comparaison" et "d'échange" » in Amacker (dir.), Présence de Saussure, Droz, Genève, pp. 55-71.

SAUSSURE, Ferdinand de 1916 : Cours de linguistique générale, Lausanne, Paris, Payot.

- 1968 : Cours de linguistique générale, édition critique par Rudolf Engler, tome 1, Wiesbaden, Otto Harrassowitz.

\section{NOTES}

1. Une « sémantique formelle ", au sens où l'entend Prieto, est une sémantique qui ne concerne nullement la langue mais seulement le «système d'intercompréhension » des locuteurs en interaction langagière.

2. Il succédait à ce poste à Henri Frei. Il fut auparavant professeur de linguistique aux Universités de Cordoba et d'Alger, puis maître de conférences à l'Université de Paris VIIIVincennes, où il enseignait la sémiologie.

3. Quelques allusions éparses à Tarski, Grize, Marx ou Althusser ne sauraient constituer un intertexte pour l'analyste.

4. Exception faite pour Éric Buyssens, qui appartient à la même génération et au même état d'esprit - celui d'un linguiste aventurier - que Prieto.

5. L'usage des guillemets de précaution est d'ailleurs récurrent chez Prieto ; ainsi par exemple, "vrai » et «faux» codes, "bonne» et "mauvaise " communication, le «vouloir dire» de l'émetteur d'un message sont des expressions qui se situent au cœur de la pensée de Prieto, mais aucune explication n'est fournie sur la nécessité de les entourer de guillemets. C'est un des problèmes que pose cette théorie : elle ne veut pas entrer dans le débat philosophique. 
6. La notion de dimension est en outre proche de celle, moins bien définie, de classe, que Prieto a utilisé dans Pertinence et pratique.

7. Les barres obliques désignent des « signifiants » ou leurs équivalents ; les guillemets anglais, des « signifiés » ou leurs équivalents.

8. À certains égards seulement, il est vrai, ainsi que nous allons le préciser plus loin.

9. Prieto a pu, cependant, dans des travaux antérieurs, marquer la prévalence de la caractéristique sur la dimension (i.e. la «classe ») : «Les notions de base ne sont donc pas pour nous celle de "classe » ni celle d' » identité », mais la notion de "différence » et son corrélat la notion de «caractéristique ", dont les premières dérivent.» (1975: 83). À moins que le sémiologue n'établisse pas le même ordre de présupposition que le locuteur, ce dont on envisage mal la possibilité, il y a entre ces deux propositions, écrites à treize ans de différence, un total renversement.

10. Cf. 1996 : 83. L'univers de discours y est rebaptisé, en suivant Umberto Eco, encyclopédie.

11. À moins que nous échappe cette subtilité qui consisterait à «ne pas indiquer une présence » sans mettre en cause cette présence même.

12. Ces trois applications sont celles effectuées dans Messages et signaux.

13. Ceci est une autre façon de marquer mon désaccord avec ce qu'a écrit Capt-Artaud: les Saussuriens ne sont pas tous sémiologues, loin s'en faut, et les discussions sur les traits pertinents ne relèvent pas seulement de la sémiologie. Il n'y a donc pas lieu, selon moi, de considérer que l'article de Prieto puisse être l'aboutissement de telles discussions, tout simplement parce que, si aboutissement il y a, celui-ci ne peut qu'être multiple.

\section{RÉSUMÉS}

Dans «Caractéristique et dimension » (1988), Luis J. Prieto propose une définition sémiologique de la syntaxe. La présente étude est consacrée à son examen. Dans un premier temps, on situe cette définition dans son contexte théorique, en l'articulant notamment avec les définitions du «sens " et de l'« univers de discours » et en mettant en avant ses conditions épistémologiques. Dans un second temps, cette même définition est portée à la critique, en fonction du principe de cohérence théorique et en considération d'autres options théoriques, proposées par L. Hjelmslev. Enfin, dans un troisième temps, on tente de montrer en quoi cette définition est sémiologique, c'est-à-dire quelle est la fonction assignée par Prieto à la sémiologie par rapport à la linguistique d'où dérive évidemment la notion de syntaxe.

«Characteristic and dimension» (1988), by L. J. Prieto, offers a 'semiological' definition of what 'syntax' is. Here we propose to examine this definition in three steps: 1) in regards to its theoretical context and in connection with the definitions of 'meaning' and 'discursive universe'; 2) through a comparative analysis between its theoretical foundations and Hjelmslev's contributions to epistemology; 3) in questioning its 'semiological' status. In the light of these observations, we would like to show how precisely Prieto put semiology and linguistics in relation each other. 


\section{AUTEUR}

SÉMIR BADIR

Chargé de recherches du F.N.R.S., Université de Liège, Semir.Badir@ulg.ac.be 